\title{
Comparison of a novel micro-assay for insulin autoantibodies with the conventional radiobinding assay
}

\author{
H.E. Naserke ${ }^{1}$, N. Dozio $^{2}$, A.-G. Ziegler ${ }^{3}$, E. Bonifacio ${ }^{2}$ \\ ${ }^{1}$ Institute of Diabetes Research, Munich, Germany \\ ${ }^{2}$ Istituto Scientifico San Raffaele, Milan, Italy \\ ${ }^{3}$ Institute of Diabetes Research and Academic Hospital Schwabing, Munich, Germany
}

\begin{abstract}
Summary Measurement of insulin autoantibodies (IAA) with a novel micro radiobinding assay which requires only $20 \mu \mathrm{l}$ of serum was compared with that in a conventional radiobinding assay which uses $600 \mu \mathrm{l}$ of serum. IAA were measured with both assays in samples from 94 new onset insulin-dependent diabetes mellitus (IDDM) patients, 97 control subjects, and 48 first degree relatives of IDDM patients selected for having IAA in the conventional radiobinding assay. Overall, 227 (95\%) of 239 samples tested were concordant, and IAA levels correlated well $\left(r^{2}=0.7\right)$ between the two assays. Discordant results were obtained in 7 new onset patients, 4 control subjects,
\end{abstract}

and 1 first degree relative, and these had low IAA levels in the respective assays. Sensitivity and specificity in the new onset IDDM patients and control subjects were $69 \%$ and $98 \%$ for the micro radiobinding assay and $72 \%$ and $98 \%$ for the conventional radiobinding assay. The use of the micro radiobinding assay should greatly facilitate islet related antibody screening, particularly in children. [Diabetologia (1998) 41: 681-683]

Keywords Insulin autoantibodies, radiobinding assay, prediction, insulin-dependent diabetes mellitus.
Insulin autoantibodies (IAA) are markers of the preclinical phase of insulin-dependent diabetes mellitus (IDDM) [1]. Their measurement, together with that of islet cell antibodies (ICA), antibodies to glutamic acid decarboxylase (GAD), and the protein tyrosine phosphatase-like antigen IA-2, forms the basis of current strategies for predicting future onset of IDDM [2]. IAA are particularly important when determining IDDM risk since their prevalence is significantly elevated in subjects developing the disease in childhood [3], and moreover, they are often the first autoantibodies to be detected [4].

Received: 8 October 1997 and in final revised form: 26 January 1998

Corresponding author: Dr. E. Bonifacio, Department of Medicine 1, Istituto Scientifico San Raffaele, via Olgettina 60, I-20132 Milan, Italy.

Abbreviations: IAA, Insulin autoantibodies; IDDM, insulindependent diabetes mellitus; GAD, glutamic acid decarboxylase; RBA, radiobinding assay; ICA, islet cell antibodies; $\mathrm{ROC}$, receiver operating characteristic.
Methodologies for the detection of IAA are based upon the original radiobinding assay (RBA) of Kurtz et al. [5], the two established and validated assays being that described by Palmer [6] and that of Soeldner [7]. These methods are sensitive but require relatively large serum volumes of up to $600 \mu$ l. This limits the use of IAA in screening when capillary blood samples are obtained and when repeat testing is necessary or desired. Recently, Williams et al. described a novel RBA based on protein-A binding, rather than polyethylene glycol (PEG) precipitation of antibody bound insulin, which requires only $20 \mu \mathrm{l}$ of serum [8]. In this study we compared results and performances of the novel micro RBA of Williams with those of the conventional RBA of Soeldner for the measurement of IAA in a large series of samples from new onset IDDM patients, control subjects and first degree relatives of IDDM patients. We report that the novel micro RBA has similar sensitivity and specificity to the Soeldner assay and should, therefore, facilitate high throughput screening, especially in young children. 


\section{Subjects and methods}

Subjects. Sera were obtained from a total of 94 IDDM patients at onset of the disease. All patients were diagnosed either at the Academic Hospital München-Schwabing, Germany or at the San Raffaele Hospital, Milan between 1993 and 1997. All were less than 20 years of age (median age 11 years; range 1-20 years); 51 were male. Sera were also obtained from 97 control subjects with no first degree family history of IDDM. The median age of control subjects was 21 years (range 2-48 years); 46 were male. Additional serum samples from 48 first degree relatives of IDDM patients in the Munich IDDM family study were included. These were selected on the basis of having IAA levels above the $99^{\text {th }}$ centile of control subjects on at least 2 occasions when tested in the conventional RBA in Munich. Relatives had a median age of 11 years (range 2-59 years); 24 were male.

IAA measurement. All samples were tested in Munich using the conventional RBA based on the Soeldner assay [7] and in Milan using a method based on the novel micro RBA of Williams [8]. For the conventional RBA results were expressed as nanounits bound insulin specifically precipitated per $\mathrm{ml}$ of serum $(\mathrm{nU} / \mathrm{ml})$. The threshold and $99^{\text {th }}$ centile of results in control subjects was calculated as $49 \mathrm{nU} / \mathrm{ml}$. This assay achieved $100 \%$ sensitivity and specificity in the Immunology of Diabetes Workshops proficiency exchanges. The micro RBA of Williams was modified by precipitating immune complexes with $50 \mu \mathrm{l}$ of $50 \mathrm{~mm}$ Tris HCI pH 8 with $1 \%$ v/v Tween 20 , $10 \%$ v/v pre-swollen protein A-sepharose (Pharmacia Biotech AB, Uppsala, Sweden) and $5 \%$ v/v pre-swollen protein Gsepharose (Pharmacia Biotech AB). The results were converted into arbitrary units by use of a standard curve. The threshold and $99^{\text {th }}$ centile of local control subjects was calculated at greater than 4.4 IAA units.

Statistical analysis. Correlation between the results obtained with the conventional RBA and the micro RBA was examined with scatterplots and linear regression analysis. Agreement between assays was assessed using Cohen's kappa measure. Sensitivity and specificity were analysed as receiver-operating characteristic (ROC) plots.

\section{Results}

Correlation between assays. A comparison of results obtained in each assay for all samples tested is shown in Figure 1. Samples from $63(67 \%)$ of 94 new onset patients and 47 of the 48 first degree relatives were detected above the respective thresholds in both assays. Samples from $24(26 \%)$ new onset patients and $93(96 \%)$ of 97 control samples were detected below these thresholds in both assays. The micro RBA detected elevated IAA levels in 2 control subjects (5.1 and 24.9 IAA units, aged 20 and 16 years) and in 2 new onset patients (4.5 and 8.7 IAA units, aged 4 and 17 years) which were not detected by the conventional RBA, and did not detect IAA in samples from 1 first degree relative aged 18 years who had $96 \mathrm{nU} /$ ml IAA in the conventional RBA and 5 new onset patients who had 50, 56, 97, 98 and $140 \mathrm{nU} / \mathrm{ml}$ IAA in the conventional RBA (aged 11, 16, 19, 13 and 6 years). The conventional RBA also detected IAA

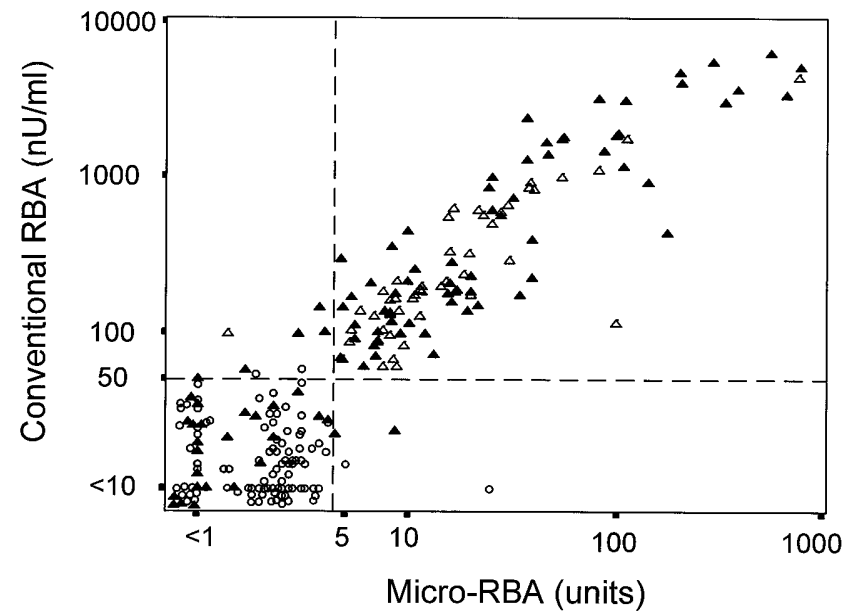

Fig. 1. Comparison of IAA results obtained in the novel micro RBA with the conventional RBA for 97 control subjects $(\bigcirc)$, 94 new onset IDDM patients $(\boldsymbol{\Delta})$ and 48 first degree relatives with IAA when determined in the conventional RBA $(\triangle)$. Thresholds for positivity for each assay are indicated as a broken line. Regression analysis gave an $r^{2}$ of $0.70\left(p<10^{-5}\right)$

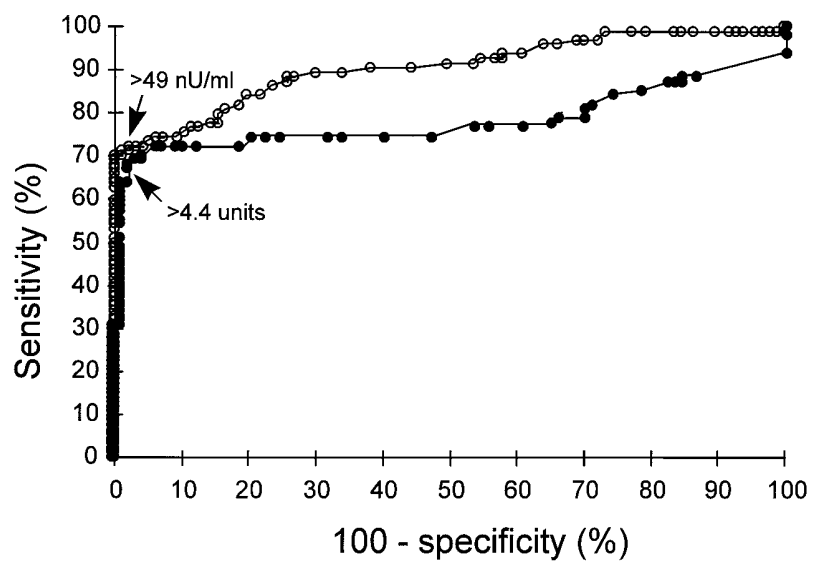

Fig. 2. ROC plots of IAA results for samples from 94 new onset IDDM patients and 97 control subjects using the novel micro RBA (O) and conventional RBA $(\bigcirc)$. Points corresponding to the previously calculated assay thresholds are indicated

in two control subjects $(53$ and $59 \mathrm{nU} / \mathrm{ml}$, aged 3 and 8 years) which were not detected in the micro RBA. Overall, $95 \%$ of samples were concordant, and agreement as assessed by Cohen's kappa measurement was 0.89 . Regression analysis gave an $r^{2}$ of 0.70 $\left(p<10^{-5}\right)$ when all samples were examined, and an $r^{2}$ of $0.68\left(p<10^{-5}\right)$ when samples from the control subjects were excluded from the analysis.

Sensitivity and specificity. ROC plots for each assay are shown in Figure 2. Using previously established thresholds corresponding to the $99^{\text {th }}$ centile of control subjects, the sensitivity in new onset IDDM patients was $69 \%$ for the micro RBA and $72 \%$ for the conventional RBA; specificities were $98 \%$ for both assays. 


\section{Discussion}

IAA are important markers of pre-clinical IDDM and there has been substantial effort in the development of assays which detect IAA in a sensitive and disease-specific manner. Until recently this has been considered possible only by using relatively large serum volumes. In this study, we report that IAA measurement with a novel micro RBA requiring only $20 \mu \mathrm{l}$ of serum was highly concordant with that of a conventional RBA which uses $600 \mu \mathrm{l}$ of serum, and achieved high sensitivity (69\%) and high specificity $(98 \%)$ when sera from new onset IDDM patients and control subjects were tested.

Overall concordance and correlation between the methods was remarkable. The few discrepant samples had relatively low IAA levels, but the majority with IAA between 50 and $100 \mathrm{nU} / \mathrm{ml}$ in the conventional RBA were also detected in the micro RBA. The quantity of IAA measured in each assay also correlated well. This is relevant since IDDM risk is associated with the amount of serum IAA as measured in the conventional assays [9]. Concordance appeared to be greater than that recently reported between the micro RBA and the assay of Palmer [8]. Whilst only 34 samples with IAA were compared in that study, more than half were discrepant. Sensitivity in new onset IDDM cases was also substantially higher in this study. Both the micro RBA and the conventional RBA yielded sensitivities of around $70 \%$ for new onset patients under 20 years of age, while in the previous study sensitivity in this age group was $52 \%$. The increased concordance and sensitivity may be due to the larger number of samples tested and the use of both protein A- and protein G- sepharose to capture antibody bound insulin as previously suggested [8].

The availability of this micro RBA should greatly facilitate screening for IDDM risk. There are currently numerous studies which include large scale screening for islet autoantibodies in the assessment of IDDM risk, the recruitment of subjects for the evaluation of intervention therapies for IDDM prevention, or for the understanding of the natural history of autoimmunity associated with diabetes. Moreover, several of these commence screening during the neonatal period when sampling is relatively difficult and would be facilitated by using capillary blood samples. Measurement of IAA with conventional assays using large serum volumes has meant that not all subjects can be screened for IAA and that capillary blood samples cannot be used. As a compromise, strategies have been proposed which include screening for antibodies to GAD and IA-2 as a first step followed by measurement of IAA and/or ICA in those with elevated antibody levels. This appears to be effective when screening is performed in older individuals, but is perhaps insufficient for screening children since it is evident that IAA are often detected earlier in life than GAD or IA-2 antibodies [4]. Their measurement in first round screening is therefore essential for early identification and intervention. The use of the micro RBA for IAA means that autoantibodies to all three antigens can be determined on less than $50 \mu \mathrm{l}$ serum and with similar assay formats. Its use will also facilitate repeat testing locally or by reference laboratories in the case of centralised registries such as ICARUS [9], allow serum samples to be stored for other determinations or studies, and enable more detailed studies of IAA binding to be performed. This assay should, therefore, markedly facilitate islet antibody screening in children.

Acknowledgements. We thank Annette Schimmel and Cristina Belloni for their help in sample preparation and antibody testing. This study forms part of the dissertation of H. E. Naserke at the Ludwig-Maximillians-University of Munich. It was supported by DLR grant FKZ:01KD9601/7and by the EU concerted action PARADIGM.

\section{References}

1. Soeldner JS, Tuttleman M, Srikanta S, Ganda OP, Eisenbarth GS (1985) Insulin-dependent diabetes mellitus and autoimmunity: islet-cell autoantibodies, insulin autoantibodies and beta cell failure. N Engl J Med 313: 893-894

2. Verge CF, Gianani R, Kawasaki E et al. (1996) Prediction of Type I diabetes in first-degree relatives using a combination of Insulin, GAD, and ICA512bdc/IA-2 autoantibodies. Diabetes 45: 926-933

3. Vardi P, Ziegler AG, Mathews JH et al. (1988) Concentration of insulin autoantibodies at onset of type 1 diabetes: inverse log-linear correlation with age. Diabetes Care 11: 736-739

4. Roll U, Christie MR, Fuechtenbusch M, Payton MA, Hawkes CJ, Ziegler AG (1996) Perinatal autoimmunity in offspring of diabetic parents: the German multicenter BABYDIAB study. Detection of humoral immune responses to islet antigens in early childhood. Diabetes 45: 967-973

5. Kurtz AB, Matthews JA, Mustaffa BE, Dagett BE, Nabarro JDN (1980) Decrease of antibodies to insulin, proinsulin and contaminating hormones after changing treatment from conventional beef to purified pork insulin. Diabetologia 18: 147-150

6. Palmer JP, Asplin CM, Clemons P et al. (1983) Insulin autoantibodies in insulin-dependent diabetes before insulintreatment. Science 222: 1337-1339

7. Vardi P, Dib SA, Tuttleman M et al. (1987) Competitive insulin autoantibody assay. Prospective evaluation of subjects at high risk for development of type 1 diabetes mellitus. Diabetes 36: 1286-1291

8. Williams AJK, Bingley PJ, Bonifacio E, Palmer JP, Gale EAM (1997) A novel micro-assay for insulin-autoantibodies. J Autoimmun 10: 473-478

9. Bingley $\mathrm{P}$ for the ICARUS Group (1996) Interactions of age, islet cell antibodies, insulin autoantibodies, and firstphase insulin response in predicting risk of progression to IDDM in ICA + relatives. The ICARUS data set. Diabetes 45: $1720-1728$ 